\title{
MONITORING AND REVAMPING THE DISTRIBUTED DATA CENTRES USING SELF-POWERED WSN
}

\author{
Deepa Kadkol T \\ Department of Information Science and Engineering, T.John Institute of Technology, Bangalore. \\ kadkoldeepa10@gmail.com
}

\begin{abstract}
The measured values in power per square foot increases considerably as the data center energy densities. The IT industry that has been seen today is growing so widely, vastly and rapidly in terms of the energy consumption in every sector. Conservative estimates also indicate a higher rate or a doubling in the consumption of IT energy as years pass-by. Hence there is an increasing need to address such a scenario. Increasing attention has been driven towards the monitoring of the environmental parameters in data. Such increased interest is due to the need of high energy efficiency of cloud services. This paper presents the design and the characterization of an energy neutral embedded wireless system, prototyped to monitor perpetually environmental parameters in servers and racks. The system has been well equipped by including a module that is meant for the functionality of energy harvesting and this has been based on the thermoelectric Generators. These generators basically convert the heat dissipation from the servers for the specific application. In this paper efforts have been made to present a power conditioning circuit optimized for the designated application beginning and considering primarily from the empirical characterization of the energy harvester. The whole system has been enhanced with several sensors. Validation has been checked and ensured to check for the performance.
\end{abstract}

Keywords: Data Center Energy, Wireless Sensor, Thermo Electric Generators, Embedded Wireless Systems $* * *$

\section{INTRODUCTION}

A data center in simple terms can be defined as, "a storage area or a repository to perform the operations such as storage, management and dissemination of data which can be of the form-physical or virtual" . A data center is considered to be the brain of the computer and is also a place where all the details are stored. Data centers are commonly run by large companies or government agencies. However, they are also increasingly used to provide a fastgrowing cloud solution service for private and business applications.

Data centers are used by various companies and government organizations and can be viewed as:

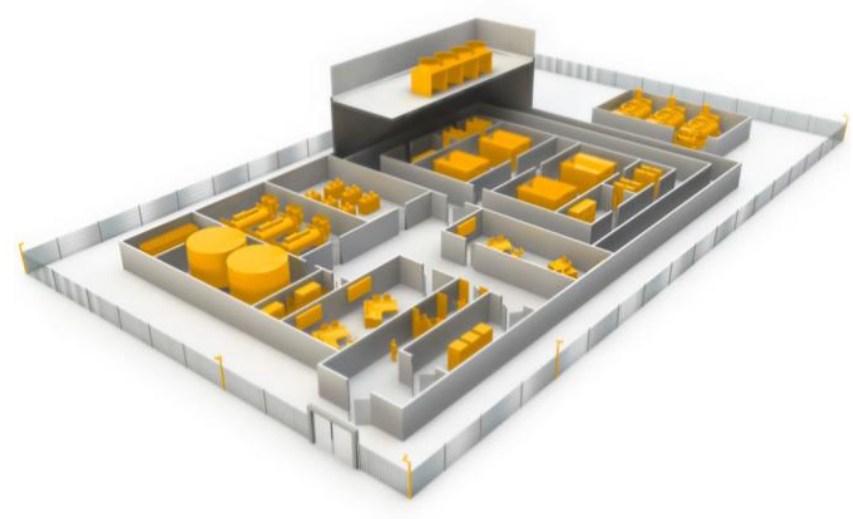

data centers are growing widely and are continuously providing significant and immense services all over the world such as in the IoT applications ,cloud and infrastructure virtualization. Days are not far when all the small to medium company-owned IT infrastructure will be replaced what can be called as the "mega-datacenters". It is quite evident that it is cost effective when these are outsourced rather than maintaining an own infrastructure and hiring managers. Now arises the question of security, reliability and maximization of service. To address these only remedy that can be considered is to rely on the automatic solutions both on cyber-security and on the physical side.

After viewing various aspects concerned with data center the major other challenges that needs to be considered for service providers is to adapt their infrastructure to a more environmentally friendly and fossil fuel free economy.

Not just the above mentioned but also abiding to the rules and regulations put forth by the governments all over the world. It is an appreciable fact that most of the companies today have put forth their renewable forms to address issues pertaining to the data centers used by the various sectors.

Hence, in order to achieve pronounced monitoring of the environment which is perhaps thermal and the energy resources and also to ensure an efficient security system it is a must and pre-requisite to design data centers and the Data Center Infrastructure Management (DCIM) in a different pattern. This setup or system can be achieved at a no cost level by utilizing the freely available power resource that is 
being provided by the environment and also by avoiding the usage of additional batteries. Among the various options or metrics used in this context the most favorable metric used is the Power Usage Effectiveness (PUE). The relation goes as- to achieve a higher efficiency the value of PUE has to be lower.

A worse utilization of the power is resulted due to additional energy used from the monitoring infrastructure. This energy increases the amount of total energy spent and thus results in the increase in the value of PUE. Proposing a solution to the above drawbacks and problems a solution in terms of various neutral network devices has been proposed in this paper.

To basically begin with what a wireless sensor technology means is that it is a network system that is capable of sending and receiving devices that provides a real-time tool to the data center operator in order to have an efficient management and observation of the space-conditioning energy. Dating back to 2010 at the Lawrence Berkeley National Laboratory an older legacy data center, a wireless sensor array and receiving devices were installed.

In this paper implementation is basically as an embedded system which concentrates on a battery less or absolutely no battery system. This is implemented in order to ensure or enable the data centers to have a system that is automated wireless.

The wasted energy which is the most abundant form of energy when the data center facilities is taken into consideration, the present system provides sampling of features such as- light, temperature etc.

The system that is currently available in the markets today are equipped with a grid. When such a system is used the data center PUE is burdened considerably.

It is found that the sampling rate is also comparatively lower than the proposed system.

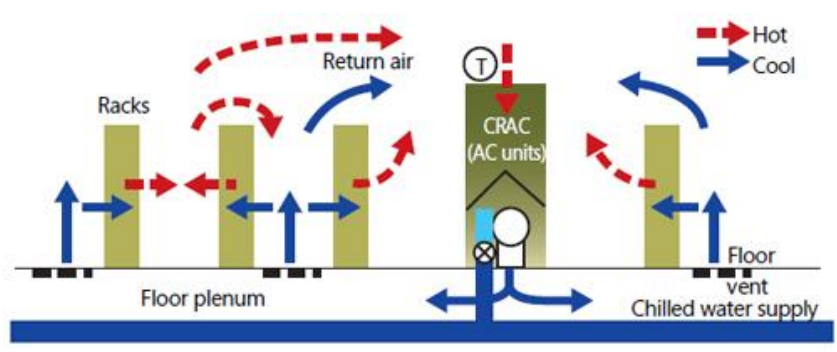

An illustration of the cross section of a data center. Cold air is blown from floor vents, while hot air rises from hot aisles. Mixed air returns to CRAC where it is cooled and cycle repeats .

The main goals in the above system is to minimize the energy consumed by CRAC and at the same time ensure no ill effect on the server

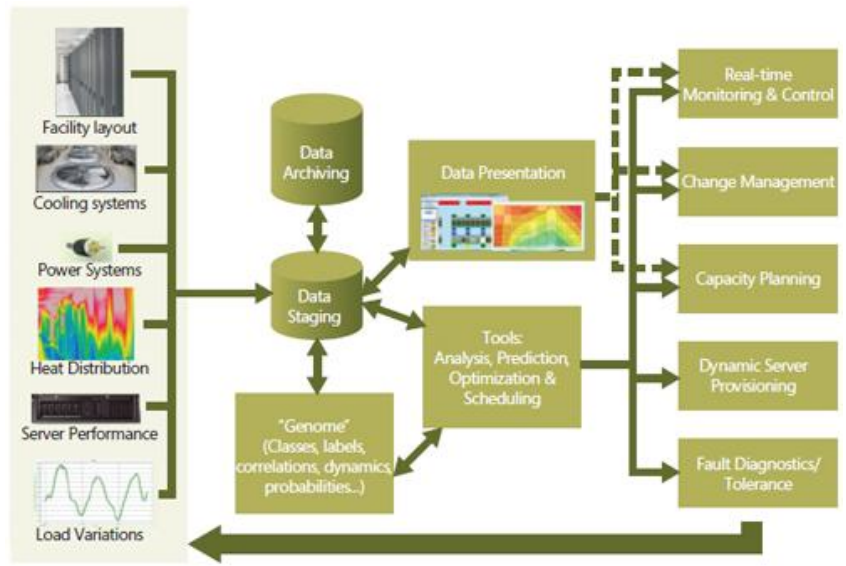

Overall architecture for the Data Center Genome system. Data collected from physical and cyber systems in data centers is correlated and analyzed to provide models and tools for data center management and performance optimization

\section{INSIGHT ON THE PROPOSED SYSTEM}

In the earlier or the present system wherein the batteries are used there is a high possibility where after a considerable time the batteries get exhausted and the only solution is to either dispose it else have a system to get it done right. Having such issues in view the proposed system in the paper has been implemented without batteries.

An added advantage in the proposed model is that the energy used is that obtained from the wasted energy and at the same time reduces the sampling time to a considerable extent.

An energy transformer named Thermoelectric Generator is built. This generator guarantees a never-ending supply. Hence the complete parameters associated with the environment can be tracked and looked on when such systems are set up in each and every CPU that is present in the data center.

The important aspect that needs to be given extensive attention is to the fact that this system that runs without batteries runs for a longer period regardless of the load that is experienced by the data center.

\section{WORKING AND IMPLEMENTATION}

When the various forecasts that have been made by various analysts are considered it has been seen that there is a higher possibility of an unimaginable increase in the thermoelectric-generation market to almost about $\$ 950$ millions by 2024 . To reach such points what is required is an integrated TEG that performs the task of scavenging watts to about hundreds or even more and at the same time would concentrate on reducing the emissions of carbon.

In any scenario wherein there is a huge loss or wastage in the thermal energy, thermoelectric scavengers can be of great use. They play a role of reusing the energy that has 
been harvested from the wasted heat. Consider an example where a major part of energy is wasted or spent in computing in the form of heat and certainly efforts are made to a considerable extent to check the equipment at a safe temperature.

Such situations where in extreme amount of heat wastage is encountered wireless sensors which reduce the cost of installation and replace batteries can be used. These sensors lower the cost of the system by boosting the heat extraction and also provide a monitoring mechanism to enforce existing DCIM systems.

Implementation details begin with:

a) Thermoelectric harvesting:

The system basically monitors the host machine and hence exploits a generator called the thermoelectric generator. This generator converts the thermal gradient of the heat into electrical energy. This conversion powers the sensors. The components of TEG include:

- Semiconductor elements

- Package to spread heat

Due to the Seebeck effect each of these elements exhibits an emf. Thus the voltage at the output is built up on account of the series connection of these elements. Final relation that can be drawn is the proportionality between the voltage generated and the thermal difference.

b) Prototype Harvester:

This consists of :

- Three stacked TEGs

- Circuit for conditioning

- Storage of harvested energy

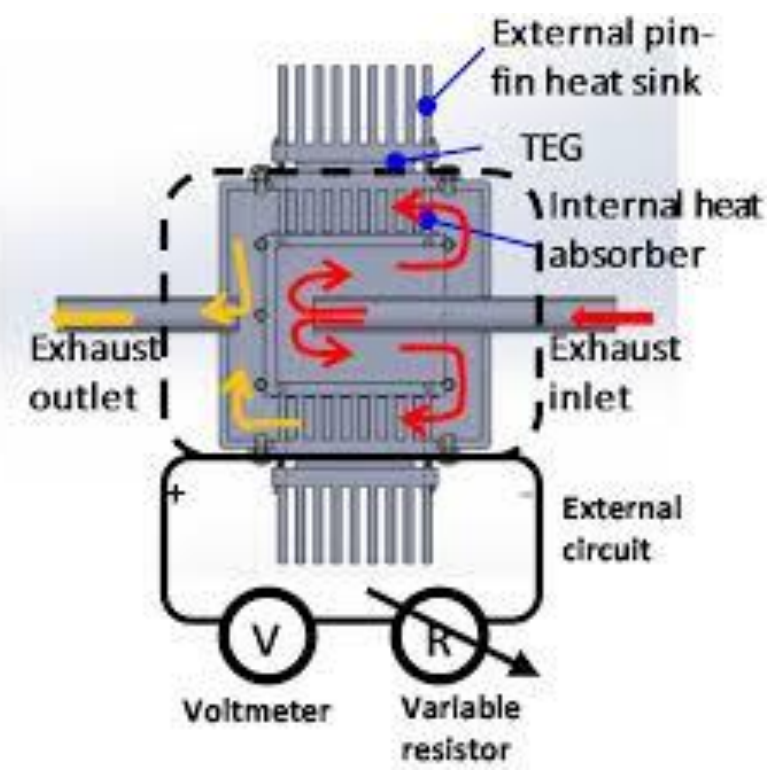

In the diagram every part is assigned and is associated with a function and is responsible to carry out those functions. The uninterrupted and continual heat flow to the TEG is ensured by the heat sink. It is to be looked on for a continuous electric current from generator and this can be achieved only when heat is removed from the cold side.
Next follows the constituents of wireless nodes. These have two circuit boards, one that houses the sensors for conditioning and storage and the next one for microcontroller and the radio chip and a thermoelectric harvester. ,

In this way ultimate goal of distributed monitoring is achieved by instrumenting the datacenter with hundreds of nodes one for each CPU or heat generating device.

c) Conditioning Hardware

Wireless sensor nodes generally require a higher power supply, but the power generated by TEG is lower than what is required by the WSN. Hence an efficient conditioning circuit to check this issue is required. The circuit should therefore raise the generated voltage up to a value that can be acceptable to feed the system and that can henceforth accumulate energy as well. Since the WSN operate with the given cycle in short bursts it is essential to provide it with a storage element that accumulates the energy and then provides it whenever required or on demand. A low amount of power is consumed hen the operation is carried under a low duty cycle. Such conditions can be supported by small energy buffers. On the contrary, a guarantee is provided by a larger storage unit regarding the operation of the nodes for longer time. This guarantee is ensured even in cases of power cuts or loss.

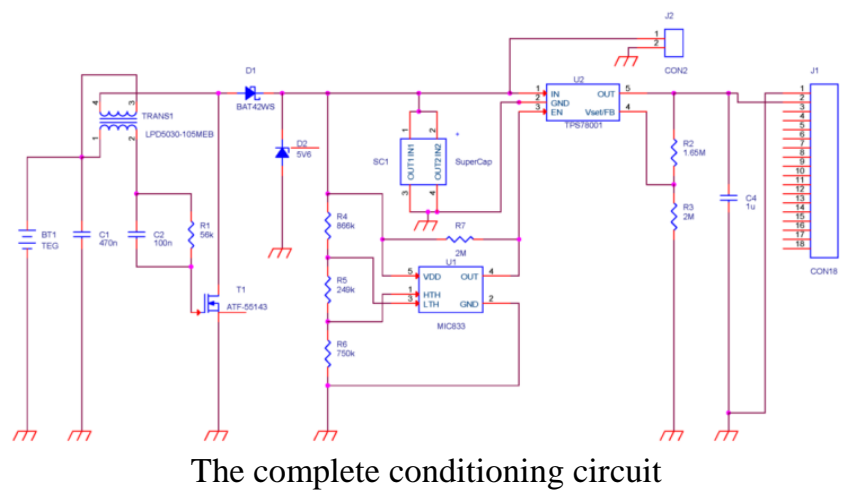

The above diagram depicts a complete conditioning system which has several components namely:

- a step-up (boost) converter

- a rectifier

- a storage unit

- a voltage comparator

- a LDO voltage regulator

The cycle begins with initiation of a resonant step-up oscillator. This oscillator converts a very low-power input voltage (hundreds of millivolts) into a higher voltage output.

Once the initial process begins, the process of filtering, decoupling and rectifying of signal is done. This signal is used by the subsequent stage. Considering the robustness required by specific applications the selection of the super capacitors are made. In this paper selection made is of a single capacitor and reason behind this is to boost recharge time rather than energy density. To maintain a steady output 
voltage The Low DropOut voltage regulator (LDO) (U2) is used. A comparator (U1) controls the LDO. The enable signal maintained by the comparator rises and LDO starts feeding the load only when accumulated charge crosses a fixed threshold. Until the charge drops which mean the value reached below threshold the enable has to placed high. Ensuring this configuration allows the output unit to be decoupled. The selection of the thresholds is based on a voltage divider that corresponds to three resistor elements: R4, R5 and R6. Adjustments of these resistors are also possible.

\section{d) Harvester Characterization}

Taking several TEGs increases the power harnessed using thermo-electric generator. These are then placed electrically in series and each TEG now represents a stage.

Now each represented stage is exposed to a fraction of the total thermal gradient, therefore it generates less electrical power. It was found that the time required by three-stage solution was the time required to recharge an empty storage capacitor. Though five-stage setup is also present it was evident that a setup of three-stage makes the perfect compromise for the specific target application. The entire paper thus focuses on the results obtained from this configuration.

Now concentrating on the characterization, the process is done directly on top of the target CPU.

It is a $\mathrm{GHz}$ dual-core ARM Cortex A15 mounted on an Arndaleboard. The power that was generated was measured. The measurement was made on the whole range of working temperatures of the processor and it was made by varying the host CPU workload.

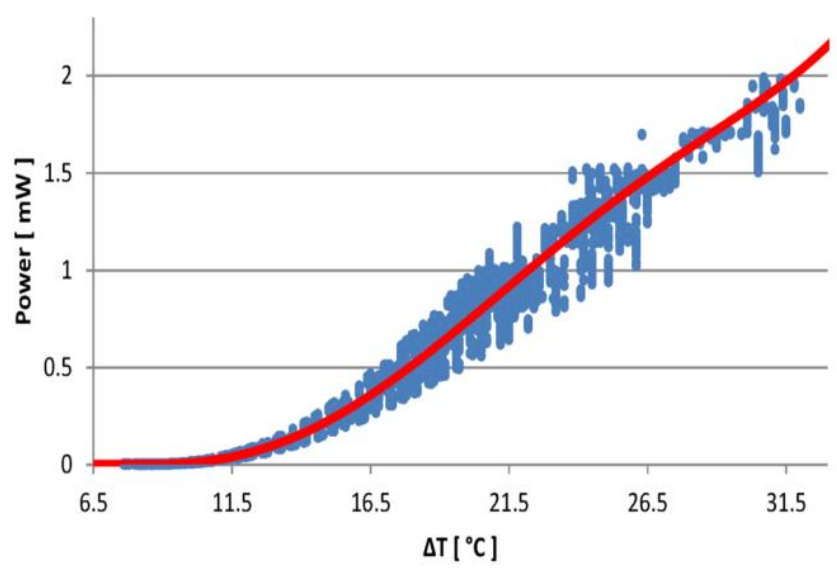

Generated output power (in Watts) versus Thermal gradient (in Celsius degrees).

The characteristic curve in red and the data set in blue on the top are all extracted from these results. On the host is an integrated temperature sensor and this measure the temperature of the ARM CPU. A thermistor is placed in between heat sink fins and this measures the temperature on the heat sink and is monitored by the WSN.
A thorough verification of those readings which was performed using a calibrated thermal imaging camera has resulted in a report as shown below:
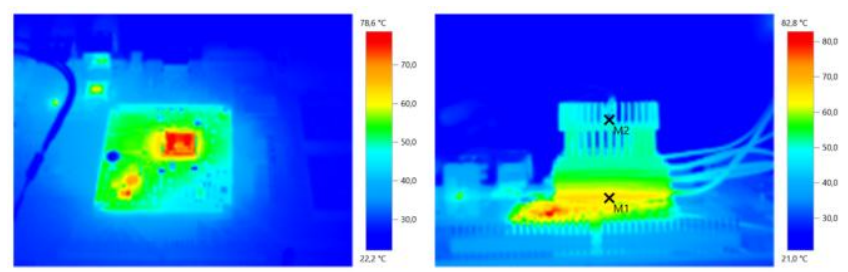

A confirmation was finally obtained about the thermal stability of the system. The obtained result shows that the thermal stability of the ARM CPU is not in any way affected by the harvesting module. On the contrary, the host is allowed to keep a maximum temperature below the warning limit of $82 \mathrm{C}$ even at its maximum clock speed ( $\mathrm{GHz}$ ) and still at a heavy $100 \%$ work load.

The next aspect to be looked upon is the controlling of the host CPU workload. During this process the host is made to run with combinations of parameters such as the clock frequency, percentage of task CPU load and also the duration. Eliminating the overhead offered by the operating system a mathematical formula can be extracted from the data that was measured.

\section{RESULTS}

It is important to check and ensure that the proposed system does not make any kind of abrupt and adverse affect on the reliability of the system. To check for this the influence of the harvester and sensor node on the thermal dissipation was evaluated. In the entire process of investigations and evaluations the temperature of the CPU was measured in three different scenarios: when there was no passive dissipation form CPU that is when it is sold by the vendor, next was with the heat sink on top and finally the entire complete prototype that was according to what was proposed. If the system causes the CPU to get heated up or not is what is to be understood from the experiment. It is also seen that the proposed system and prototype helps working of CPU at relatively lower and safer temperatures.

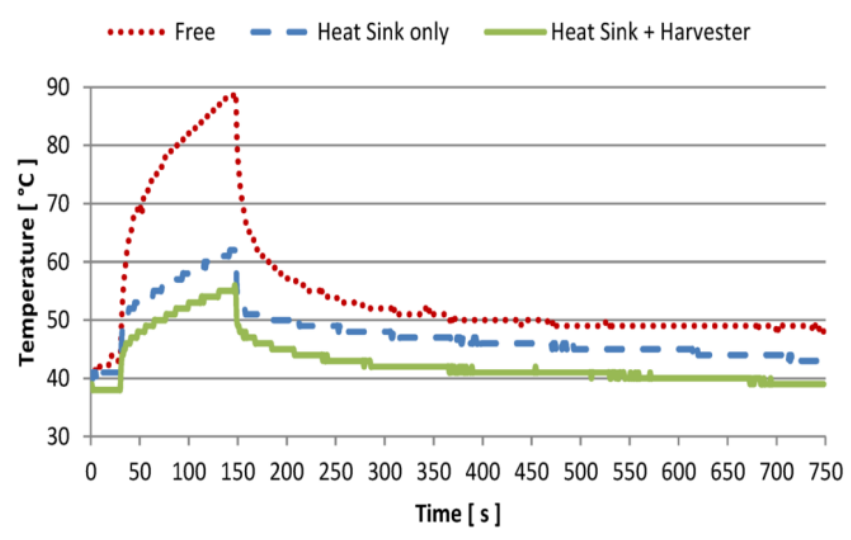

CPU temperature trend in three configuration: with and without the heat sink, and with the proposed prototype on top. 
Various effective results have been obtained to evaluate the effectiveness of the adaptive application. This has been done under varying working conditions. The figure depicts the condition that is encountered or attained when capacitor charge reaches the threshold of operation of the node.

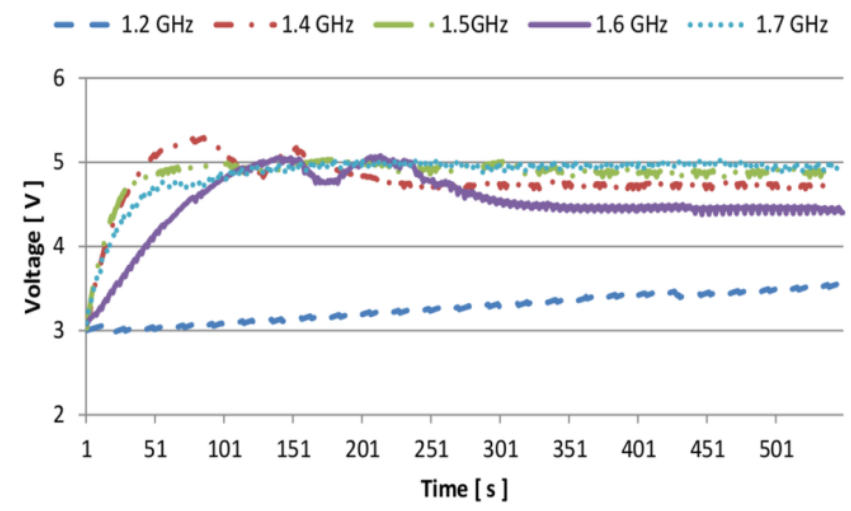

Measured super capacitor voltage using adaptive sampling rate for different clock frequencies of the host processor.

A certain initiation is required for the implementation is what is shown in the graph. The temperature readings are not accurate during this time because the heat is not propagated uniformly. This is also accompanied by regular fluctuations in the voltage but the value of the fluctuating voltage never drops below the threshold. There is an increase in the super capacitor towards a value of $5 \mathrm{~V}$ and this is only after the temperature stabilizes.

The major interest and focus in this paper has been made on experiments that involve a single server and sensor and heat sink that corresponds to the server. When a practical and realistic aspect is taken into consideration regarding a data center, several number of WSN and network resources are required. Such a complexity can result in even more complexities that becomes difficult to address. But at the same time, standing by the realistic implementation and justifying the same in this paper an expectation is to increase the energy recovery efficiency. This can possibly be done by depending on the process that is considered with automatic manufacturing. Undoubtedly significant and research in depth is required to deploy a real data center environment. But nevertheless the proposed prototype marks a significant step when efficiency, autonomy and size are considered.

\section{CONCLUSION}

In order to understand the energy usage patterns of various deployed data centers it is essential to collect cooling data and this is regarded as the first and foremost step. Only with a deeper and wider understanding about various parameters such as power consumption, network traffic and so forth a considerable reduction on the total data center energy can be achieved and this can be done without an abrupt disturbance to the user's performance.

The main aspects that have been dealt with is a self-powered WSN node that is used for the monitoring of the data center.
The design of the device has been explained covering aspects from the selection of a considerable and efficient TEG as required by the ARM CPU package. The results and the step wise methodology regarding the prototype characterization has been performed. In this process an ARM board that is a device providing a high performance has been checked on. Two aspects that are- implementation at fixed interval as well as dynamic data rate have been programmed by the prototype. The overall impact and outcome demonstrates that the proposed system is able to self-power or automate itself whenever it is placed in any environment. Various other kinds of sensors can also integrated with the proposed approach is what can be concluded.

\section{REFERENCES}

[1] Press Release. IDC Finds Growth, Consolidation, and Changing Ownership Patterns in Worldwide Datacenter Forecast. IDC Corporate, 10 November 2014.

[2] The Guardian, 8 June, 2015. G7 Leaders Agree to Phase out Fossil Fuel Use by End of Century.

[3] Piper Jaffray CIO Survey. Technology, Media \& Telecom 2015. Available online: https://piper2.bluematrix.com/sellside/EmailDocViewer?enc rypt=7856c68e-3f1a-4ce9-a7e7-99fe25145cd9\&mime=pdf

[4] Amazon.com, Inc. (Amazon Web Services). Amazon Initiatives for Sustainable Energy. http://aws.amazon.com/it/about-aws/sustainable-energy/

[5] Rodriguez, M.; Ortiz Uriarte, L.; Jia, Y.; Yoshii, K.; Ross, R.; Beckman, P. Wireless sensor network for datacenter environmental monitoring. In Proceedings of the 2011 Fifth International Conference on Sensing Technology (ICST), Palmerston North, New Zealand, 28 November-1 December 2011; pp. 533-537.

[6] Zervos, H. Energy Harvesting for Automotive Applications; IDTechEx: Cambridge, MA, USA, 2011;

[7] IDTechEx Ltd. Thermoelectric Energy Harvesting 20142024: Devices, Applications, Opportunities.2012

[8] Jayakumar, S.; Reda, S. Making Sense of Thermoelectrics for Processor Thermal Management and Energy Harvesting. In Proceedings of the International Symposium on Low Power Electronics and Design, Rome, Italy, 22-24 July 2015.

[9] Rodriguez, M.; Ortiz Uriarte, L.; Jia, Y.; Yoshii, K.; Ross, R.; Beckman, P. Wireless sensor network for datacenter environmental monitoring. In Proceedings of the 2011 Fifth International Conference on Sensing Technology (ICST), Palmerston North, New Zealand, 28 November-1 December 2011; pp. 533-537.

[10] Microsoft Corporation. Data Center Genome Project. Available online: http://research.microsoft.com/enus/projects/dcgenome/

[11] "An analysis of a large scale habitat monitoring application," Robert Szewczyk, 2nd ACM Conference on Embedded Networked Sensor Systems (SenSys 2004), Baltimore, Md., November, 2004

[12] "Energy-aware server provisioning and load dispatching for connection-intensive internet services," Gong Chen et. al, 5th USENIX Symposium on Networked 
Systems Design \& Implementation (NSDI 2008), San Francisco, Calif., April 2008.

[13] "Fidelity and yield in a volcano monitoring sensor network," Geoff Werner-Allen et. al, 7th Symposium on Operating Systems Design and Implementation (OSDI '06), Seattle, Wash., November 2006 [14] "FireWxNet: a multi-tiered portable wireless system for monitoring weather conditions in wildland fire environments," Carl Hartung et. al, 4th International Conference on Mobile Systems, Applications, and Services (MobiSys 2006), Uppsala, Sweden, June 2006 